\title{
Numerical Investigation of Near-Field Plasma Flows in Magnetic Nozzles
}

\author{
Kamesh Sankaran* \\ Whitworth University, Spokane, WA, 99251
}

\author{
Kurt A. Polzin ${ }^{\dagger}$ \\ NASA-Marshall Space Flight Center, Huntsville, AL 35812
}

\begin{abstract}
The development and application of a multidimensional numerical simulation code for investigating nearfield plasma processes in magnetic nozzles are presented. The code calculates the time-dependent evolution of all three spatial components of both the magnetic field and velocity in a plasma flow, and includes physical models of relevant transport phenomena. It has been applied to an investigation of the behavior of plasma flows found in high-power thrusters, employing a realistic magnetic nozzle configuration. Simulation of a channelflow case where the flow was super-Alfvénic has demonstrated that such a flow produces adequate 'back-emf' to significantly alter the shape of the total magnetic field, preventing the flow from curving back to the magnetic field coil in the near-field region. Results from this simulation can be insightful in predicting far-field behavior and can be used as a set of self-consistent boundary conditions for far-field simulations. Future investigations will focus on cases where the inlet flow is sub-Alfvénic and where the flow is allowed to freely expand in the radial direction once it is downstream of the coil.
\end{abstract}

\section{Nomenclature}

$\begin{array}{ll}\mathbf{B} & \text { magnetic induction, T } \\ \overline{\bar{B}}_{M} & \text { Maxwell stress tensor } \\ E & \text { electric field strength, V/m } \\ E^{\prime} & \text { electric field in the plasma frame, } \mathrm{V} / \mathrm{m} \\ \mathcal{E} & \text { energy density of the plasma, } \mathrm{J} / \mathrm{m}^{3} \\ k & \text { thermal conductivity, } \mathrm{W} / \mathrm{m} . \mathrm{K} \\ \mathbf{j} & \text { current density, } \mathrm{A} / \mathrm{m}^{2}\end{array}$

$\begin{array}{ll}p & \text { pressure, } \mathrm{Pa} \\ \mathbf{q} & \text { dissipative power per area, } \mathrm{W} / \mathrm{m}^{2} \\ T & \text { temperature, } \mathrm{K} \\ \mathbf{u} & \text { fluid velocity, } \mathrm{m} / \mathrm{s} \\ \eta & \text { resistivity, } \Omega . \mathrm{m} \\ \mu_{\circ} & \text { permeability of free space, } \mathrm{H} / \mathrm{m} \\ \rho & \text { mass density, } \mathrm{kg} / \mathrm{m}^{3}\end{array}$

\section{Introduction}

M AGNETIC nozzles are used in many plasma thrusters to convert random thermal energy into directed kinetic energy and to control the direction of the exhaust. However, due to the solenoidal nature of the magnetic field it is essential to ensure that the plasma detaches from the field, making this a problem of active research.,2 While other investigations have looked into far-field phenomena, like detachment, this paper discusses numerical simulations of the near-field behavior of plasma jets in magnetic nozzles, where the applied field gradients are greatest. The development of a multidimensional numerical tool that can be used to investigate plasma detachment mechanisms was introduced in Ref. [3]. This code solves the conservation form of the magnetohydrodynamic (MHD) equations, accounting for thermal nonequilibrium between electrons and the rest of the plasma and including appropriate models for ionization, equation of state and various transport phenomena. This is an extension of previous simulations of self-field magnetoplasmadynamic thrusters (MPDT) ${ }^{4,5}$ and a lithium Lorentz force accelerator. ${ }^{6}$

The outline for the rest of this paper is as follows. The governing equations for the nozzle simulations are described in Sect. II. The geometry of the model, including the description of the magnetic nozzle, and the boundary conditions used are discussed in Sect. III. We present the results and a discussion of a plasma simulation test case involving a super-Alfvénic channel flow in solenoidal magnetic fields in Sect. IV, and discuss the next steps of this effort in Sect. V.

*Associate Professor, Physics Department. Senior Member AIAA

$\dagger$ Propulsion Research Engineer, Propulsion Research and Technology Applications Branch, Propulsion Systems Department. Senior Member AIAA.

This material is declared a work of the U.S. Government and is not subject to copyright protection in the United States. 


\section{MHD Equations for Simulations}

The governing equations for an MHD flow problem are represented in conservation relations for mass, momentum, magnetic flux, and energy. These can be compactly written in the form,

$$
\frac{\partial}{\partial t}\left[\begin{array}{c}
\rho \\
\rho \mathbf{u} \\
\mathbf{B} \\
\mathcal{E}
\end{array}\right]+\nabla \cdot\left[\begin{array}{c}
\rho \mathbf{u} \\
\rho \mathbf{u u}+\overline{\bar{p}}-\overline{\overline{\mathcal{B}}}_{M} \\
\mathbf{u B}-\mathbf{B \mathbf { u }} \\
(\mathcal{E}+p) \mathbf{u}-\overline{\overline{\mathcal{B}}}_{M} \cdot \mathbf{u}
\end{array}\right]=\mathbf{D} \text {. }
$$

The right hand side of the equation, D, contains dissipative effects, which are also written as the divergence of fluxes. ${ }^{6}$ The compact vector-tensor equations are expanded into their individual vector components and rewritten in a finitevolume form so that they can be solved. The expanded equation set, assuming no azimuthal variation $(\partial / \partial \theta=0)$, is:

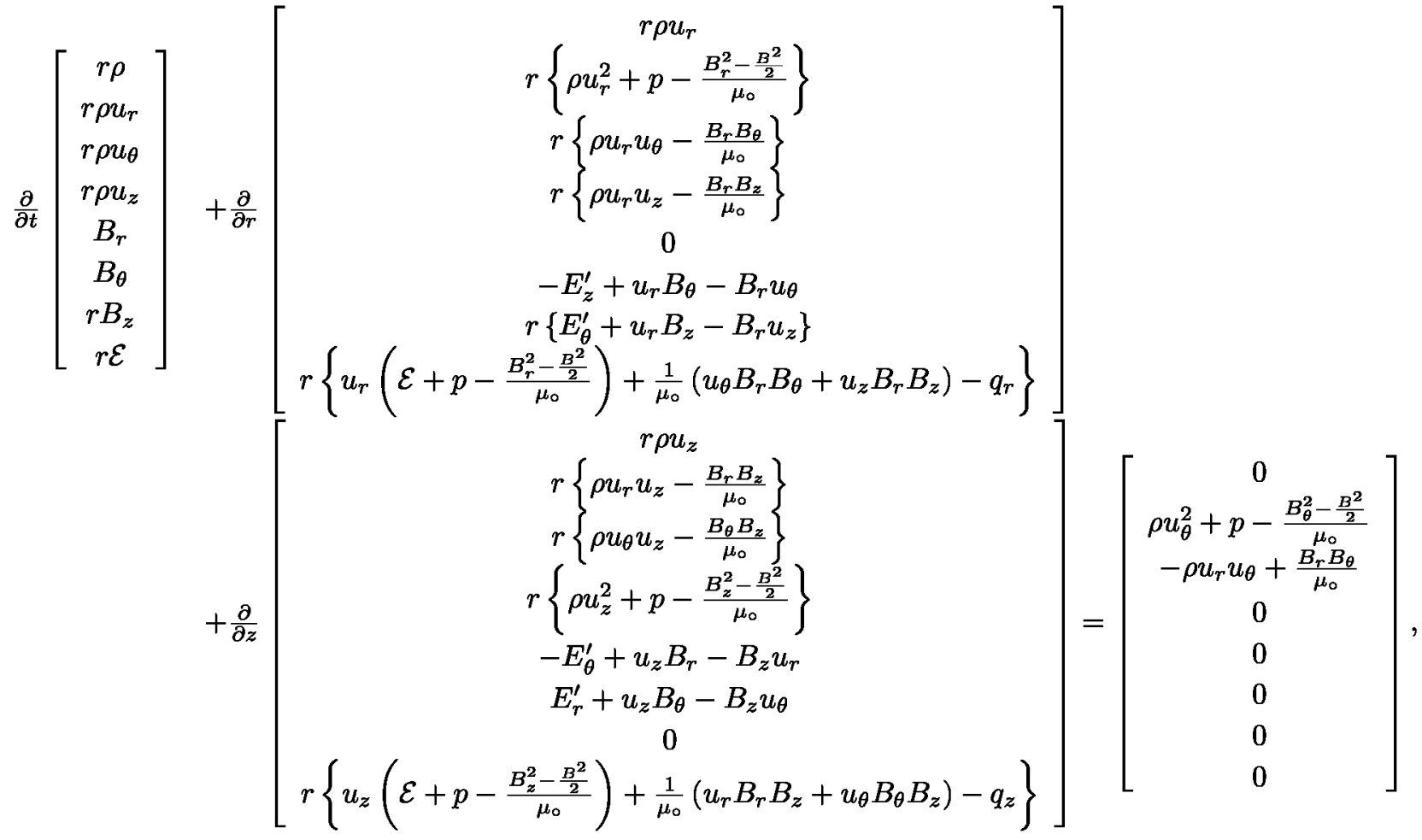

where

$$
\begin{aligned}
& q_{r}=\left(E_{z}^{\prime} B_{\theta}-E_{\theta}^{\prime} B_{z}\right) / \mu_{\circ}+\left(k_{r r} \partial T_{e} / \partial r\right)+\left(k_{r z} \partial T_{e} / \partial z\right) \\
& q_{z}=\left(E_{r}^{\prime} B_{\theta}-E_{r}^{\prime} B_{\theta}\right) / \mu_{\circ}+\left(k_{z r} \partial T_{e} / \partial r\right)+\left(k_{z z} \partial T_{e} / \partial z\right) .
\end{aligned}
$$

The thermal nonequilibrium between the electrons and the heavy species (ions of varying levels of ionization and neutrals) is accounted by maintaining a separate energy equation for the electrons. In the presence of an applied magnetic field, the components $B_{r}$ and $B_{z}$ are the sum of the steady-state applied field and an induced field that is initially time-varying but eventually converges to a steady-state value. The set of MHD equations is closed by the generalized Ohm's law,

$$
\mathbf{E}^{\prime}=\mathbf{E}+(\mathbf{u} \times \mathbf{B})=\eta \mathbf{j}+\frac{\left(\mathbf{j} \times \mathbf{B}-\nabla p_{e}\right)}{e n_{e}},
$$

and Ampère's law. The models for transport coefficients, equation-of-state, ionization and thermal nonequilibrium are described in Ref. [6]. 


\section{Simulated Geometry and Boundary Conditions}

\section{III.A. Physical Domain and Magnetostatic Model}

A separate magnetostatic solver ${ }^{7}$ is used to create an applied magnetic field topology. The magnetic coils in real experiments have tens of thousands of loops, while the coils in magnetostatic simulation had only a handful of loops. Consequently, the primary goal of our magnetostatic modeling effort was to recreate a reasonable and realistic topology. In Fig. 1a we show the nozzle topology for the experiment described by Kuriki and $\mathrm{Okada}^{8}$ generated using the magnetostatic solver.

a)

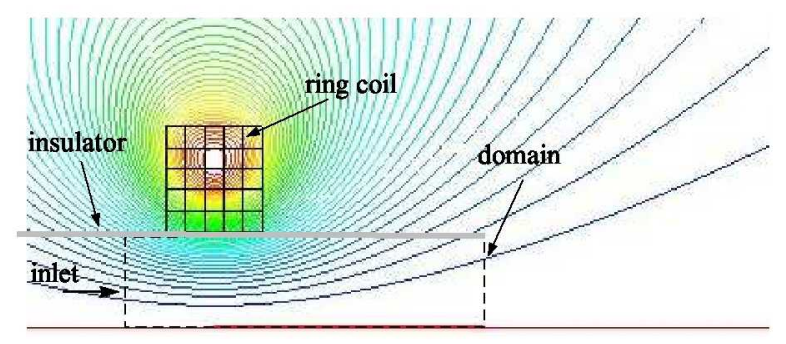

b)

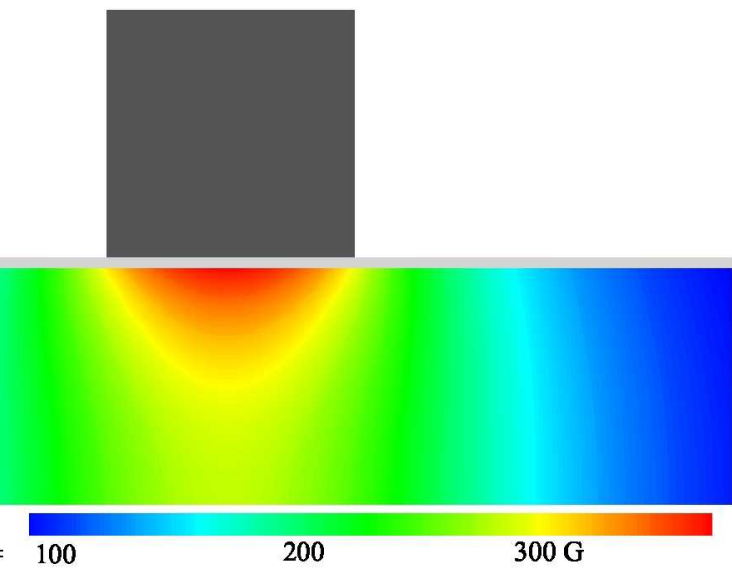

Figure 1. a) Schematic of the experimental setup (based upon Ref. [8]) and the domain that was modeled in this paper. Magnetostatic simulation results showing the magnetic flux lines in the $r-z$ plane of the nozzle are presented, with these data used as the applied field background in this simulation; b) Magnitude of the applied magnetic field produced by the coil.

The computationally-generated field was compared to published values from the experimental setup. Despite our simplifying the problem by using only a few magnet coil loops, the magnetostatic solver produced a magnetic field topology possessing field values that were within a few percent of those measured. ${ }^{8}$ The magnetostatic solver ${ }^{7}$ yields the relevant variables $\left(B_{r}, B_{z}\right)$ on an orthogonal $(r, z)$ grid. An interpolation script was used to transfer the magnetic field data onto the non-orthogonal grid used by the MHD code. Though it is not our goal to simulate the experiment in Ref. [8], our simulation uses the magnetic field topology used in that experiment because of its simplicity and appropriateness for our investigation.

The domain of the simulation is shown in Fig. 1a as the dotted rectangle. Here, the bottom surface of the coil is at a distance $R=5.0 \mathrm{~cm}$ from the centerline and has a $5.0 \mathrm{~cm}$ by $5.0 \mathrm{~cm}$ rectangular cross-section. has a thickness of 5.0 $\mathrm{cm}$ as well. The computational domain extends $5.0 \mathrm{~cm}$ upstream and $10.0 \mathrm{~cm}$ downstream from the center of the coil. The domain is bounded at the top $\left(R_{\max }=4.98 \mathrm{~cm}\right)$ by an insulator, resulting in a restricted channel-flow simulation.

\section{III.B. Boundary Conditions}

Near-field investigations in applied solenoidal fields pose special challenges for numerical simulations owing to the strong curvature of the magnetic field, large gradients in the flow parameters, and interactions with material boundaries. We proceed with a discussion of the boundary conditions used in this paper.

\section{III.B.1. Flow Properties}

- Inlet - At the inlet $(z=0)$, a specified mass flow rate of the propellant enters at a specified temperature at sonic conditions. The velocity vector is set to be parallel to the magnetic field vector at the inlet, treating the incoming plasma as fully magnetized.

- Exit - At the axial outflow boundary, the axial gradient of the flow quantities is set to be same as the axial gradient just inside the exit plane.

- Solid Walls - In the radial direction, at $R_{\max }$, the flow is obstructed by a solid surface. Here, we enforce $\mathbf{n} \cdot \mathbf{u}=0$. This surface is treated as a thermal insulator. Consequently thermal conduction is set to zero. 
- $\underline{\text { Centerline }}-$ At the axis of symmetry $(r=0)$, there are no radial convective or conductive fluxes. Moreover, there are no radial gradients here.

\section{III.B.2. Field Properties}

- Inlet and Exit - In this simulation, the computational domain is not large enough to assume that the magnetic field is zero at the boundaries. In fact, such an assumption is not justified given that one of the primary objectives of this investigation is to study the interplay between the plasma and the magnetic nozzle. Therefore, the magnetic field in the ghost cells adjacent to the inlet and the exit consists of both the applied field of the magnetic nozzle and the induced field produced by internal plasma currents. Contributions from the latter are self-consistently calculated for each ghost cell (at the general location $\left(r_{g}, z_{g}\right)$ ) using the Biot-Savart law, summing the contributions from all the currents inside the domain, ${ }^{9}$

$$
\mathbf{B}_{\text {ind }}\left(r_{g}, z_{g}\right)=\int_{\mathcal{V}} \frac{\mu_{\circ}}{4 \pi} \frac{\mathbf{j} \times \mathbf{r}}{r^{3}} d \mathcal{V} \text {. }
$$

While more efficient ways for implementing this can be found in Ref. [10], we employ an explicit numerical evaluation of Eq. (4).

- Solid Walls - At the insulated boundary at $R_{\max }$, the magnetic field is assumed to diffuse instantaneously into the wall (i.e., $\eta \rightarrow \infty$ ) implying that the surface current is zero.

- Centerline - At the axis of symmetry $j_{r}=j_{\theta}=0$, and the inductive component of the electric field is zero because $u_{r}=u_{\theta}=B_{r}=B_{\theta}=0$. The axial current density, $j_{z}$ can be obtained from the magnetic field at the point adjacent to $r=0$ using a Taylor series approximation, which yields

$$
\left.j_{z}\right|_{r=0}=\frac{\left.4 B_{\theta}\right|_{\frac{\Delta r}{2}}}{\mu_{\circ} \Delta r} .
$$

\section{Results and Analysis}

Theories of plasma detachment from magnetic fields ${ }^{11,12}$ identify the ratio of the flow speed to the Alfvén speed, $v_{A}=B / \sqrt{\mu_{\mathrm{o}} \rho}$, as a key parameter in this process. This ratio, also known as the Alfvén Mach number $M_{A}$, corresponds to the square root of the ratio of the kinetic energy of the plasma to the magnetic field energy which, in equilibrium, scales with the square root of the plasma beta,

$$
M_{A}=\frac{u}{v_{A}}=\frac{u}{B / \sqrt{\mu_{\circ} \rho}}=\sqrt{\frac{\rho u^{2} / 2}{B^{2} /\left(2 \mu_{\circ}\right)}} \simeq \sqrt{\frac{p}{B^{2} /\left(2 \mu_{\circ}\right)}}=\sqrt{\beta} .
$$

We chose to examine a channel flow case where the flow speed exceeds the Alfvén speed to analyze the how the flow and the field shape each other. Therefore we set $u / v_{A}=M_{A}=2.0$, based on a magnetic field value of $301 \mathrm{G}$. This corresponds to the value of the initial applied field under the center of the coil $(z=5 \mathrm{~cm})$ taken at the radial location $r=3.4 \mathrm{~cm}$, which corresponds to the radius where half the mass in a uniform flow would be below this radius and half above. The mass flow rate was fixed at $\dot{m}=6.0 \mathrm{~g} / \mathrm{s}$, and the temperature at the inlet was set at $T_{e}=T_{h}=1.5 \mathrm{eV}$. These parameters determine the density and the speed of the incoming plasma flow. The plasma conditions chosen are representative of those found in propulsive flow exhausts. For a domain that extends $15 \mathrm{~cm}$ in the axial dimension, a plasma flow of $u_{z} \simeq 3000 \mathrm{~m} / \mathrm{s}$ takes $\mathcal{O}(100) \mu \mathrm{s}$ to reach establish equilibrium.

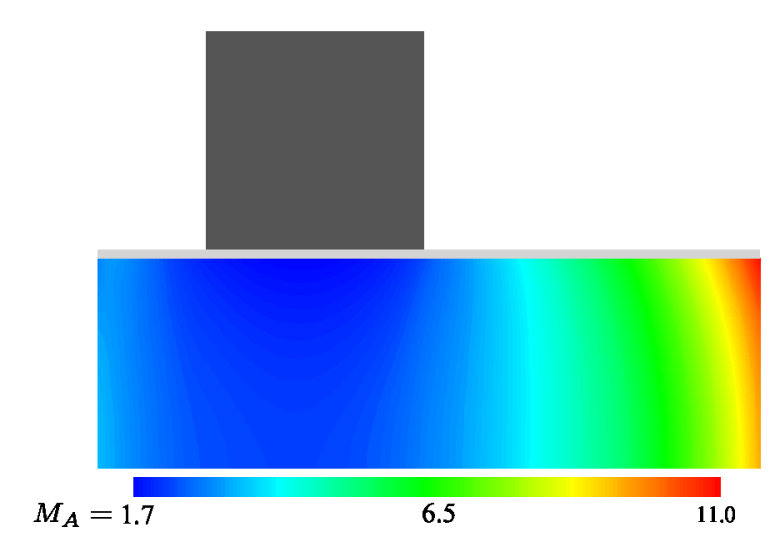

Figure 2. Profile of the Alfvén Mach number, $M_{A}$, obtained for a converged, steady-state solution.

Fig. 2 shows the ratio of the flow speed to the Alfven speed at the end of the calculation. Since the flow is set to be super-Alfvénic at the region of strongest magnetic field, 
directly below the coil, $M_{A}$ is even higher in the regions where the magnetic field is weaker. Under these conditions the magnetic Reynolds number $R e_{m} \sim \mathcal{O}(1)$, indicating that convective effects are significant, and that the 'back emf' will play an important role in this problem. This is consistent with the results of this simulation. With $M_{A}>1$, the induced field shields out the applied field. This is primarily accomplished by large azimuthal currents that are induced in the plasma. As shown in Fig. 3, $j_{\theta}$ is large below the corners of the coil where the gradients in the applied magnetic field are the strongest. The large azimuthal current in a thin layer near the inlet is an artifact of the boundary counditions, due to which the incoming flow encounters the magnetic field and begins to swirl.

Because $\operatorname{Re}_{m} \sim \mathcal{O}(1)$, dissipative effects are also significant. Consequently, the magnetic field is not completely shielded from the interior of the plasma, with some of the field diffusing inwards. In Fig. 4, flux tubes of the initial (applied) and the final (applied + induced) magnetic field are presented, showing how the plasma acts to shape the total magnetic field. Due to the large azimuthal currents induced in opposite directions at the edges of the coil, shown in Fig. 3, the magnitude of the axial magnetic field between the bounds of the coil is very low, and the field downstream of the coil is significantly altered by the plasma.

The final field is stretched axially at the exit, as shown in Fig. 4, allowing the plasma to exit the domain without closing back to the coil in the near-field region. The angle made by the initial (applied) and final (applied

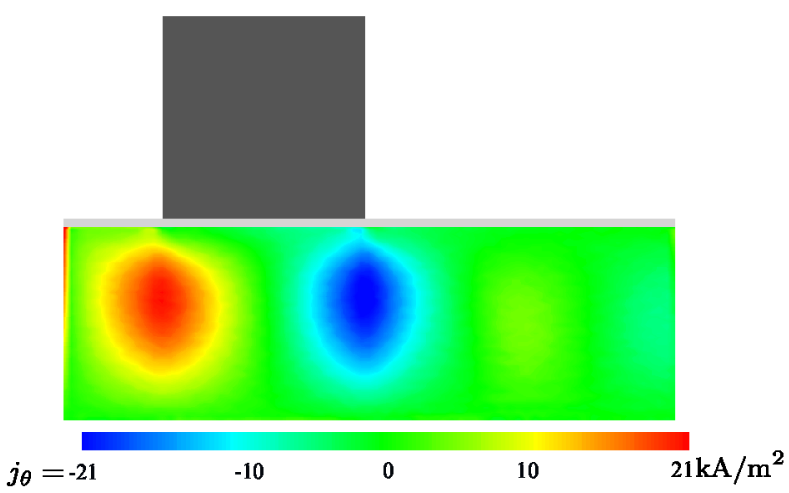

Figure 3. Azimuthal plasma currents, $j_{\theta}$, calculated within the domain. + induced) fields with the $\hat{z}$ axis can be a useful metric to understand the effect of the plasma flow in shaping the magnetic field. As shown in Fig. 5, the axial flux of the plasma out of the domain is not seriously impeded by the applied magnetic field. In fact, in this case the field and plasma align themselves with each other quite well, with both vectors pointing primarily in the axial direction at the exit.

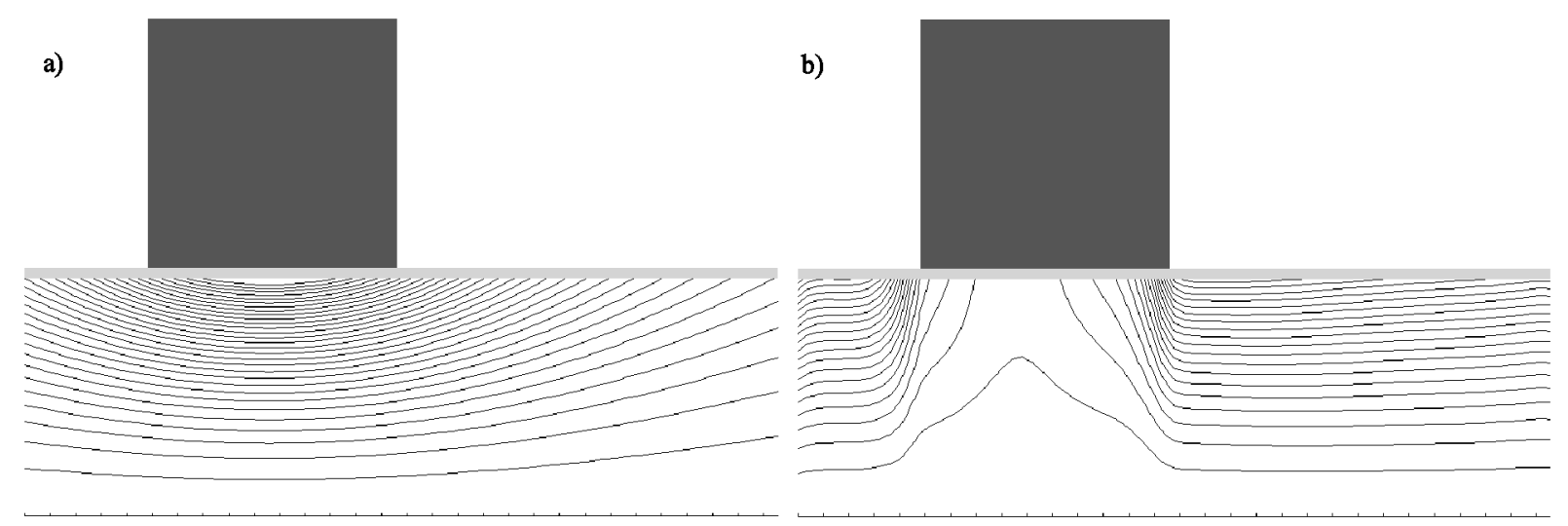

Figure 4. Magnetic flux tubes of a) the initial (applied) magnetic field and b) the final (total) magnetic field, showing the effect of a super-Alfvénic plasma on the field distribution.

\section{Concluding Remarks}

We have presented the development and application of a code that calculates the time-dependent evolution of all three spatial components of magnetic field and velocity of plasma flows in magnetic nozzles. We have used it to investigate the behavior of a plasma flow, with plasma parameters typically found in high-power thrusters, in a magnetic nozzle configuration used in an experiment. We simulated a channel-flow case where the flow was superAlfvénic, and demonstrated that such a flow produces adequate 'back-emf' to shape the total field and prevent the flow from curving back to the magnetic coil in the near-field region. We showed that this is accomplished through the production of large azimuthal currents, especially below the coil corners where the applied field gradients are largest. 


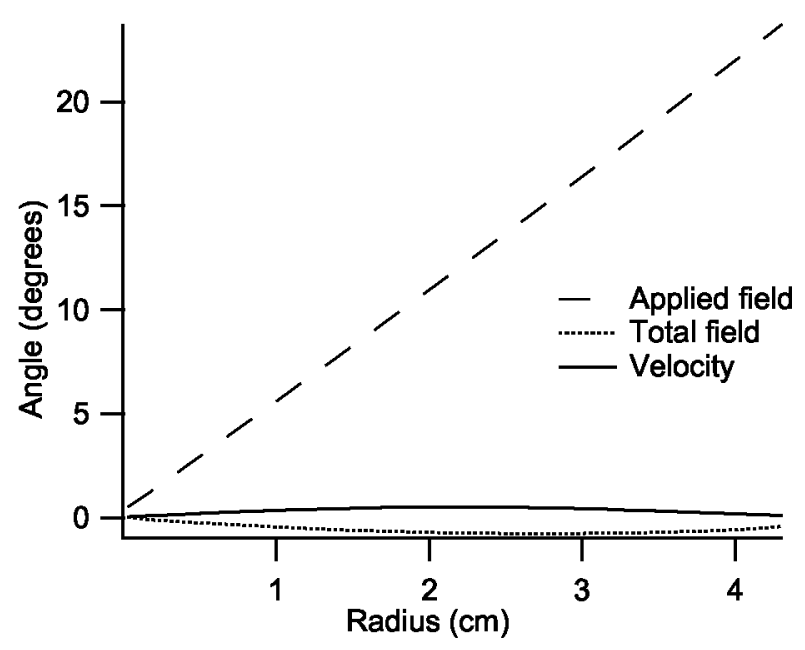

Figure 5. The angle made by the initial (applied) and final (applied + induced) fields with the $\hat{z}$ axis at the exit plane.

In the future, we intend to simulate cases where the inlet flow is sub-Alfvénic to compare with the results of the present simulation, and also allow unimpeded radial expansion of the plasma jet downstream of the coil.

Given the limitations of the continuum model used in this work, we have restricted our investigation to the nearfield region of the magnetic nozzle. Other investigations, such as that found in Ref. [1], discuss the far-field region. Nevertheless, observations of plasma behavior in the near-field of the magnetic nozzle have proved to be informative and the results from this simulation can be extrapolated to estimate far-field behavior and can yield self-consistent boundary conditions for far-field simulations.

\section{Acknowledgments}

The authors thank Marc Rollins for his assistance in calculating the applied field produced by the coil. Support for Prof. Sankaran was provided by the Washington Space Grant and Whitworth University.

\section{References}

${ }^{1}$ B. N. Breizman, M. R. Tushentsov, and A. V. Arefiev. "Magnetic nozzle and plasma detachment model for a steady-state flow". Physics of Plasmas, 15:057103, 2009.

${ }^{2}$ C. A. Deline et al. "Plume detachment from a magnetic nozzle". Physics of Plasmas, 16:033502, 2009.

${ }^{3}$ K. Sankaran and K. A. Polzin. "Development of Numerical Tools for the Investigation of Plasma Detachment from Magnetic Nozzles". AIAA-2007-4386, 2007.

${ }^{4}$ K. Sankaran, L. Martinelli, S.C. Jardin, and E.Y. Choueiri. "A Flux-Limited Numerical Method for the MHD Equations to Simulate Propulsive Plasma Flows". Int. J. Num. Meth. Eng., 53(5):1415-1432, 2002.

${ }^{5}$ K. Sankaran, E.Y. Choueiri, and S.C. Jardin. "Comparison of Simulated Magnetoplasmadynamic Thruster Flowfields to Experimental Measurements". Journal of Propulsion and Power, 21:129-138, 2005.

${ }^{6} \mathrm{~K}$. Sankaran. "Simulation of Plasma Flows in Self-Field Lorentz Force Accelerators". PhD thesis, Princeton U., 2005.

${ }^{7}$ Ansoft Corporation. "Maxwell User Guide". http://www.ansoft.com/, 2002.

${ }^{8} \mathrm{~K}$. Kuriki and O. Okada. "Experimental Study of a Plasma Flow in a Magnetic Nozzle". Physics of Fluids, 13:2262, 1970.

${ }^{9}$ J. Cassibry and P. G. Mikellides. Personal communication. 2008.

${ }^{10}$ S. C. Jardin. Computational Methods in Plasma Physics. CRC Press, 2010.

${ }^{11}$ E. N. Parker. "Dynamics of the Interplanetary Gas and Magnetic Fields". Astrophysical Journal, 128:664, 1958.

${ }^{12}$ A. V. Arefiev and B. N. Breizman. "Magnetohydrodynamic scenario of plasma detachment in a magnetic nozzle". Physics of Plasmas, 12:043504, 2005. 